\title{
Fluorescent Mueller-matrix polarimetry of biological tissues in differentiation of benign and malignant tumors
}

\author{
Yu.O. Ushenko ${ }^{1}$, I.I. Lakusta ${ }^{1}$, O.I. Olar ${ }^{2}$, O.Yu. Novakovska ${ }^{2}$ \\ ${ }^{I}$ Chernivtsi National University, Correlation Optics Department, \\ 2, Kotsyubinsky str., 58012 Chernivtsi, Ukraine \\ ${ }^{2}$ Bukovinian State Medical University, 58000 Chernivtsi, Ukraine \\ E-mail:a.dubolazov@chnu.edu.ua
}

\begin{abstract}
The work is aimed at investigation of diagnostic efficiency provided by a new azimuthally stable Mueller-matrix method for analyzing laser fluorescence coordinate distributions characterizing biological tissue histological sections. A new model of generalized optical anisotropy of biological tissues containing protein networks has been proposed in order to ascertain the processes of laser autofluorescence. The influence of complex mechanisms of both phase anisotropy (linear birefringence and optical activity) and linear (circular) dichroism was taken into account. The interrelations between the azimuthally stable Mueller-matrix elements characterizing laser autofluorescence and different mechanisms of optical anisotropy have been determined. The statistic analysis of coordinate distributions of these Mueller-matrix rotation invariants has been offered. Thereupon the quantitative criteria (statistic moments of the 1st to 4th orders) of differentiation of histological sections of uterus wall tumor - group 1 (dysplasia) and group 2 (adenocarcinoma) have been estimated.
\end{abstract}

Keywords: fluorescent biopsy, biological tissues, malignant tumors of uterus wall.

Manuscript received 06.11.14; revised version received 24.02.15; accepted for publication 27.02.15; published online 08.06.15.

\section{Introduction}

Biological tissues represent structurally heterogeneous optical anisotropic media with absorption. To describe interactions of polarized light with these complex systems more generalized approximations are required, for example those based on Mueller-matrix formalism. Nowadays, many practical techniques including measurements and analysis of Mueller matrices corresponding to the investigated samples are applied in biological and medical researches [1-5]. A separate direction - laser polarimetry - was formed in matrix optics in recent 10-15 years [6-10].

In parallel to polarimetric methods, the fluorescence ones are being actively developed. These methods are grounded on the diagnostic use of fluorescence effects in protein molecules and their complexes. Consequently, the valid results concerning the cancer fluorescence diagnostic of cavitary organs were obtained [11-16].

In this research, the model of generalized optical anisotropy inherent to the tissues of women's reproductive sphere is suggested, and on its basis the method of Mueller-matrix mapping of laser polarization autofluorescence of histological sections taken from biopsy of benign (dysplasia) and malignant (adenocarcinoma) tumors of uterus wall was applied.

\section{Brief theory}

In this work, we have limited ourselves by considering the spectral-selective case - luminescence of optically 
active porphyrins of biological tissue in red $\left(\lambda_{f}=\right.$ $0.63 \ldots 0.65 \mu \mathrm{m})$ spectral range [17-31]. Excitation of autofluorescence was realized using a blue solid-state laser with the wavelength $\lambda=0.405 \mu \mathrm{m}$ that coincides with the porphyrin absorption peak.

The following model ideas concerning optical anisotropy of protein networks are used as the basis for the description of laser polarization autofluorescence of biological tissues.

The formation of laser polarization autofluorescence is based on the mechanisms of optically anisotropic absorption (linear and circular dichroism) [18]; fluorescence of porphyrin molecules ("linear" oscillators) and generated by them networks ("elliptical" oscillators) [19] and mechanisms of phase anisotropy (linear and circular birefringence) that modulate the fluorescent radiation of protein molecules and their structures. The mentioned scenario can be described by using Mueller-matrix formalism.

2.1. Absorption - Amino acids and polypeptide chains (primary structure of protein) made by them form the fibrillar (secondary structure) protein networks possessing the linear dichroism. Optical manifestations of such a mechanism are exhaustively described by the next Mueller matrices:

$$
\begin{aligned}
& \{\Psi\}=\left\|\begin{array}{cccc}
1 & \varphi_{12} & \varphi_{13} & 0 \\
\varphi_{21} & \varphi_{22} & \varphi_{23} & 0 \\
\varphi_{31} & \varphi_{32} & \varphi_{33} & 0 \\
0 & 0 & 0 & \varphi_{44}
\end{array}\right\|, \text { where } \\
& \varphi_{i k}= \begin{cases}\varphi_{12}=\varphi_{21}=(1-\Delta \tau) \cos 2 \rho, \\
\varphi_{13}=\varphi_{31}=(1-\Delta \tau) \sin 2 \rho, \\
\varphi_{22}=(1+\Delta \tau) \cos ^{2} 2 \rho+2 \sqrt{\Delta \tau} \sin ^{2} 2 \rho, \\
\varphi_{23}=\varphi_{32}=(1-\Delta \tau) \sin 2 \rho, \\
\varphi_{33}=(1+\Delta \tau) \sin ^{2} 2 \rho+2 \sqrt{\Delta \tau} \cos ^{2} 2 \rho, \\
\varphi_{44}=2 \sqrt{\Delta \tau} .\end{cases}
\end{aligned}
$$

Here, $\quad \Delta \tau=\frac{\tau_{x}}{\tau_{y}}, \quad\left\{\begin{array}{l}\tau_{x}=\tau \cos \rho \\ \tau_{y}=\tau \sin \rho\end{array}, \quad \tau_{x}, \quad \tau_{y}\right.$ absorption coefficients of linearly polarized orthogonal components of the light beam amplitude.

Availability of complex spiral-like structures or their combinations (third-order structure) of polypeptide protein structures forms the circular dichroism. Optical manifestations of these configuration peculiarities are characterized by the Mueller matrix:

$$
\{\Phi\}=\left\|\begin{array}{cccc}
1 & 0 & 0 & \phi_{14} \\
0 & \phi_{22} & 0 & 0 \\
0 & 0 & \phi_{33} & 0 \\
\phi_{41} & 0 & 0 & 1
\end{array}\right\| \text {, where }
$$

$\phi_{i k}=\left\{\begin{array}{l}\phi_{22}=\phi_{33}=\frac{1-\Delta g^{2}}{1+\Delta g^{2}}, \\ \phi_{14}=\phi_{41}= \pm \frac{2 \Delta g}{1+\Delta g^{2}} .\end{array}\right.$

Here, $\quad \Delta g=\frac{g_{\otimes}-g_{\oplus}}{g_{\otimes}+g_{\oplus}}, \quad g_{\otimes}, \quad g_{\oplus} \quad$ are absorption indices of left- $(\otimes)$ and right-hand $(\oplus)$ circularly polarized components of light beam amplitude.

2.2. Fluorescence - Polarization appearance of porphyrin fluorescence is characterized by Muellermatrix provided in [20]

$\{F\}=\left\|\begin{array}{cccc}1 & F_{12} & 0 & 0 \\ F_{21} & F_{22} & 0 & 0 \\ 0 & 0 & F_{33} & 0 \\ 0 & 0 & 0 & F_{44}\end{array}\right\|$, where

$F_{i k}=F_{11}^{-1}\left\{\begin{array}{l}F_{11}=a-b \sin ^{2} \vartheta, \\ F_{12}=F_{21}=-b \sin ^{2} \vartheta, \\ F_{22}=b\left(1+\cos ^{2} \vartheta\right), \\ F_{33}=2 b \cos \vartheta, \\ F_{44}=2 c \cos \vartheta .\end{array}\right.$.

Here, $\theta$ is the scattering angle; $a$ and $b$ are the interrelated constants for the system of linear oscillators in isotropic medium, which are defined by the following relations

$$
\begin{aligned}
& a=0.5\left(1+\left\langle\cos ^{2} \varepsilon\right\rangle\right), \\
& b=0.25\left(3\left\langle\cos ^{2} \varepsilon\right\rangle-1\right),
\end{aligned}
$$

where $\varepsilon$ is the angle between the emission dipole and the polarization azimuth of the exciting beam. There are two experimentally important cases where the theoretical limits of $\left\langle\cos ^{2} \varepsilon\right\rangle$ can be predicted [20]: if the emission and absorption dipoles are collinear $\left\langle\cos ^{2} \varepsilon\right\rangle=\frac{3}{5}$, and if the emission dipoles are randomly aligned $\left\langle\cos ^{2} \varepsilon\right\rangle=\frac{1}{3}$

The parameter $c$ is undoubtedly related to optical activity. From the classical viewpoint, the "circular oscillator" would be caused by the induced electric and magnetic transition dipole moments being fully parallel or antiparallel and having the same magnitude. Following this description, optically active liquid-crystal molecules are "elliptical oscillators". The limiting values of $c$ for this system would be $c=\frac{5}{16}$.

2.3. Phase modulation of fluorescence. Amino acids and polypeptide chains made by them (primary structure of protein) demonstrate optical activity and are characterized by the following matrix operator 
$\{\Omega\}=\left\|\begin{array}{cccc}1 & 0 & 0 & 0 \\ 0 & \omega_{22} & \omega_{23} & 0 \\ 0 & \omega_{32} & \omega_{33} & 0 \\ 0 & 0 & 0 & 1\end{array}\right\|$, where

$\omega_{i k}=\left\{\begin{array}{l}\omega_{22}=\omega_{33}=\cos 2 \gamma, \\ \omega_{23}=-\omega_{32}=\sin 2 \gamma .\end{array}\right.$

Here, $\gamma$ is the rotation angle of polarization plane fluorescent radiation.

Fibrillar (secondary structure) protein networks formed by polypeptide chains possess linear birefringence and are exhaustively described by the Mueller matrix

$$
\begin{aligned}
& \{D\}=\left\|\begin{array}{llll}
1 & 0 & 0 & 0 \\
0 & d_{22} & d_{23} & d_{24} \\
0 & d_{32} & d_{33} & d_{34} \\
0 & d_{42} & d_{43} & d_{44}
\end{array}\right\|, \text { where } \\
& d_{i k}=\left\{\begin{array}{l}
d_{22}=\cos ^{2} 2 \rho+\sin ^{2} 2 \rho \cos \delta, \\
d_{23}=d_{32}=\cos 2 \rho \sin 2 \rho(1-\cos \delta), \\
d_{33}=\sin ^{2} 2 \rho+\cos ^{2} 2 \rho \cos \delta, \\
d_{24}=-d_{42}=\sin 2 \rho \sin \delta, \\
d_{34}=-d_{43}=\cos 2 \rho \sin \delta, \\
d_{44}=\cos \delta .
\end{array}\right.
\end{aligned}
$$

Here, $\rho$ is the direction of fibril's package, $\delta$ phase shift between linearly-polarized orthogonal components of fluorescent light amplitude.

Considering the effect of all mechanisms of optically anisotropic absorption and phase anisotropy, the Mueller matrix of laser polarization autofluorescence of the biological tissue protein network can be written as follows

$$
\{M\}=\{D\}\{\Omega\}\{F\}\{\Psi\}\{\Phi\}=\left\|\begin{array}{cccc}
1 & M_{12} & M_{13} & M_{14} \\
M_{21} & M_{22} & M_{23} & M_{24} \\
M_{31} & M_{32} & M_{33} & M_{34} \\
M_{41} & M_{42} & M_{43} & M_{44}
\end{array}\right\| .
$$

The analysis of matrix (8) shows that elements $M_{i k}$ characterize superposition of mechanisms of linear $\Delta \tau$ and circular $\Delta g$ dichroism; fluorescence of linear $F_{12 ; 21 ; 22 ; 33}$ and elliptical $F_{44}$ oscillators with the following phase modulation of this radiation by optically active molecules $\theta$ and birefringent $\delta$ networks of them.

The "information content" of matrix elements is different. The set of elements $M_{i=1 ; k=1 ; 2 ; 3 ; 4}\left(F_{12}\right)$ characterizes fluorescence of linear oscillators originated from the anisotropic absorption. The elements $M_{i=2 ; 3 ; k=1 ; 2 ; 3 ; 4}\left(F_{21 ; 22 ; 33}\right)$ define phase-modulated $(\delta, \theta)$ fluorescence of linear oscillators. Finally, the values of elements $M_{i=4 ; k=1 ; 2 ; 3 ; 4}\left(F_{21 ; 22 ; 33}, F_{44}\right)$ consist of complex information concerning fluorescence of linear $\left(F_{21 ; 2 ; 33}\right)$ and elliptical $\left(F_{44}\right)$ oscillators in optically anisotropic medium with linear and circular birefringence.

It should be noted that not all elements of matrix (8) are suitable for practical usage. The reason for that consists in azimuthally dependence of most matrix elements - in general, 12 of 16 elements change at rotation of the sample around the probing axis. It is shown in $[2,4]$ that the following elements of the matrix $\{M\}$, as well as their combinations, are azimuthally stable, independent of the rotation angle $\Theta$ of the sample

$$
\left\{\begin{array}{l}
M_{11}(\Theta)=\text { const }, \\
M_{14}(\Theta)=\text { const }, \\
M_{41}(\Theta)=\text { const }, \\
M_{44}(\Theta)=\text { const }
\end{array}\right.
$$

The analysis of the above presented rotation invariants (9) shows that by measuring

$$
\left\{\begin{array}{r}
M_{14}=W\left(F_{12}(a, b), \varphi_{i k}(\Delta \tau), \phi_{i k}(\Delta g)\right) \\
M_{41}=Q\left(F_{21 ; 22 ; 33}(a, b), F_{44}(c), \varphi_{i k}(\Delta \tau),\right. \\
\left.\phi_{i k}(\Delta g), d_{i k}(\delta), \omega_{i k}(\theta)\right)
\end{array}\right.
$$

it is possible to obtain separated and azimuthally-stable information about laser polarization fluorescence of linear $\left(F_{12 ; 21 ; 22 ; 33}\right)$ and elliptical $\left(F_{44}\right)$ oscillators excited by the mechanisms of linear and circular dichroism of laser radiation in optically anisotropic biological tissue.

\section{Analysis and discussion of experimental results}

Optically thin (the geometric thickness $d=30 \mu \mathrm{m}$, extinction coefficient $\tau<0.1)$ histological sections of postsurgical biopsy of uterus wall tumors related to two types were used as objects of investigation:

- $\quad$ benign tumor (dysplasia) - group 1 (21 samples);

- malignant tumor (adenocarcinoma) - group 2 (21 samples).

Histological sections were prepared according to the standard technique on the freezing microtome.

The measurements of coordinate distributions of Mueller-matrix elements characterizing polarization properties of histological sections of uterus wall tumors were performed in the setup of the standard Stokespolarimeter. The detailed description of the optical scheme and basic parts of experimental setup was presented in a series of articles [21-28]. In this research, for autofluorescence excitation we used a diode laser with the wavelength $\lambda=405 \mu \mathrm{m}$ and power $W=50 \mu \mathrm{W}$. For spectral division of polarization fluorescence in front of CCD-camera, an interference filter was placed with the maximal transmission bandpass $\lambda_{f}=0.63 \ldots 0.65 \mu \mathrm{m}$, which corresponded to intensity of laser polarization autofluorescence maximally achieved under these conditions. 
The values of Mueller-matrix rotation invariants (relations (10), (11)) were determined by means of the algorithm

$$
\left\{\begin{array}{l}
M_{14}=S_{1}^{\otimes}-0.5\left(S_{1}^{0}+S_{1}^{90}\right), \\
M_{41}=0.5\left(S_{4}^{0}+S_{4}^{90}\right) .
\end{array}\right.
$$

Here, $S_{i=1 ; 4}^{0 ; 90 ; \otimes}$ are the Stokes vector parameters in the points of digital image of laser polarization autofluorescence of histological sections measured for a series of linearly $\left(0^{\circ}, 90^{\circ}\right)$ and right-hand $(\otimes)$ circularly polarized probing laser beams within the limits of 2D $(m \times n)$ ensemble of light-sensitive pixels.

For objective assessment of coordinate distributions of Mueller-matrix rotation invariants $q \equiv\left\{M_{14 ; 41}(m \times n)\right\}$, we used the traditional method of statistic analysis. The set of statistic moments of the $1^{\text {st }}$ to $4^{\text {th }}$ orders that characterize distributions $q$ was calculated using the following algorithms

$$
\begin{aligned}
& Z_{1}=\frac{1}{N} \sum_{j=1}^{N}|q|_{j}, Z_{2}=\sqrt{\frac{1}{N} \sum_{j=1}^{N}(q)_{j}^{2}}, \\
& Z_{3}=\frac{1}{Z_{2}^{3}} \frac{1}{N} \sum_{j=1}^{N}(q)_{j}^{3}, \quad Z_{4}=\frac{1}{Z_{2}^{4}} \frac{1}{N} \sum_{j=1}^{N}(q)_{j}^{4} .
\end{aligned}
$$

where $N$ is the number of pixels of CCD-camera.

Figs 1 and 2 present the series of experimentally measured (relations (10)) spectral-selective $\left(\Delta \lambda_{f}\right)$ Mueller-matrix fluorescent images $M_{14 ; 41}(m \times n)$ characterizing laser polarization fluorescence of optically anisotropic histological sections of uterus wall tumor of the group 1 (Fig. 1) and group 2 (Fig. 2).

The analysis of the data obtained shows the common regularity - the non-zero value of all the Mueller matrix elements characterizing polarization fluorescence of histological sections of uterus wall tumor. This fact experimentally confirms the model structure of Mueller-matrix (8) as superposition of

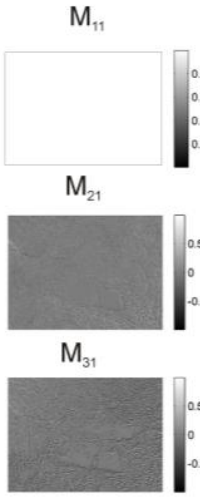

$\mathrm{M}$

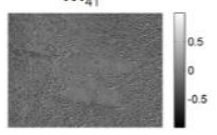

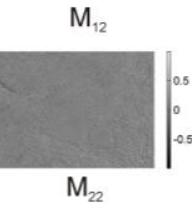

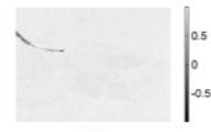

$\mathrm{M}_{32}$

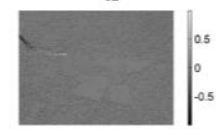

$\mathrm{M}_{42}$

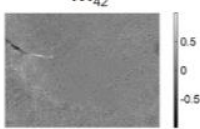

$M_{13}$

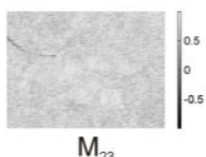

$\mathrm{M}_{23}$

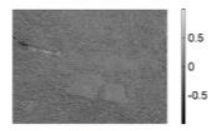

$M_{33}$

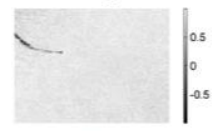

$\mathrm{M}_{43}$

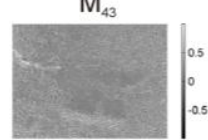

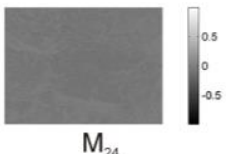

$\mathrm{M}_{24}$

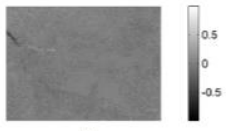

$\mathrm{M}_{34}$

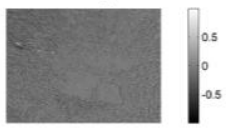

$\mathrm{M}_{4}$

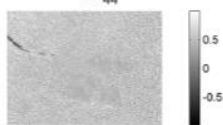

Fig. 1. Mueller-matrix fluorescent images of histological sections of benign (dysplasia) tumor of uterus wall (group 1).

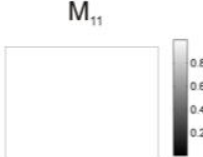

$\mathrm{M}_{21}$

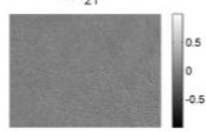

$\mathrm{M}_{31}$

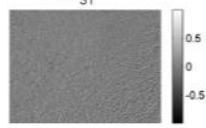

$M_{4}$

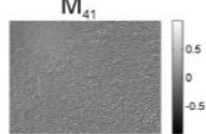

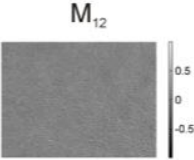

$\mathrm{M}_{22}$

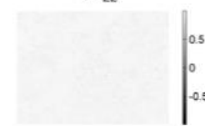

$\mathrm{M}_{32}$

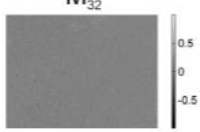

$\mathrm{M}_{42}$

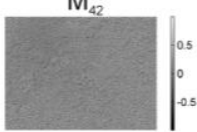

$\mathrm{M}_{13}$

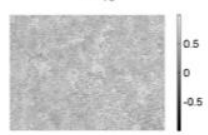

$\mathrm{M}_{23}$

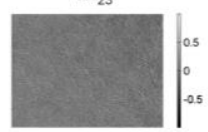

$M_{33}$

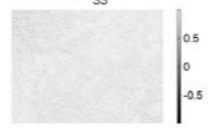

$\mathrm{M}_{4}$

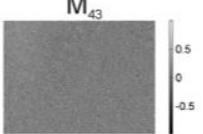

$\mathrm{M}_{14}$

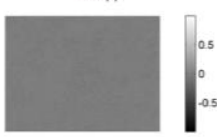

$\mathrm{M}_{24}$

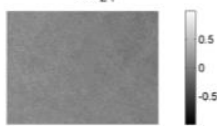

$M_{34}$

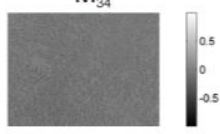

$M_{44}$

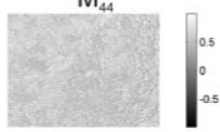

Fig. 2. Mueller-matrix fluorescent images of histological sections of malignant (adenocarcinoma) tumor of uterus wall (group 2).

(C) 2015, V. Lashkaryov Institute of Semiconductor Physics, National Academy of Sciences of Ukraine 
matrix operators, which characterize linear and circular dichroism (Eqs. (1), (2)), fluorescence of porphyrins (Eqs. (3)-(5)) and phase modulation of this radiation ((Eqs. (6), (7)). However, like it was assumed in the model analysis, polarization autofluorescence is the most vividly manifested in coordinate distributions of Mueller-matrix rotation invariants $M_{41}$ and $M_{14}$ for the samples of the group 1 and group 2. These distributions $(100 \times 100$ pix $)$ are illustrated by the series of coordinate dependences presented in Figs. 3 and 4.

The analysis of coordinate distributions of invariant $M_{14}$ (Fig. 3), which characterizes the processes of transformation of circularly polarized fluorescent radiation into the linearly one, reveals more than two times drop in the value $\left(M_{14} \downarrow\right)$ and range of changes ( $\left.\Delta M_{14} \downarrow\right)$ of this parameter in the plane of tissue histological section with adenocarcinoma (parts 3 and 4).

The inverse situation occurs for Mueller-matrix invariant $M_{41}$ (Fig. 4), which characterizes the processes of transformation of linearly polarized fluorescent radiation into a circular one.

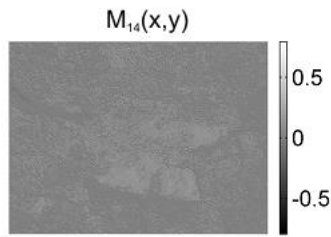

(1)

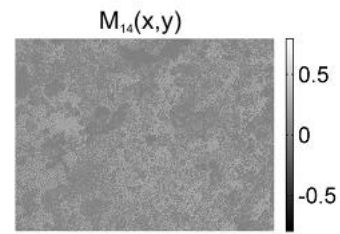

(2)
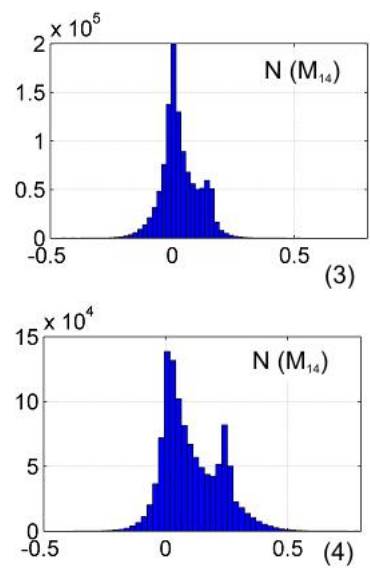

Fig. 3. Coordinate distributions of the Mueller-matrix rotation invariant $M_{14}$ for optically thin histological sections of postsurgical biopsy of benign (fragments (1), (3)) and malignant (fragments (2), (4)) tumor of uterus wall.

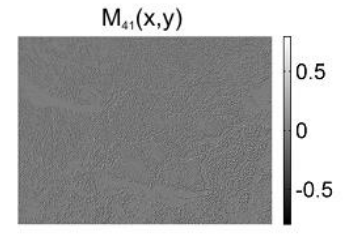

(1)

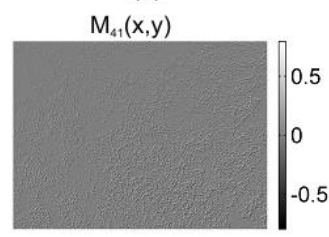

(2)

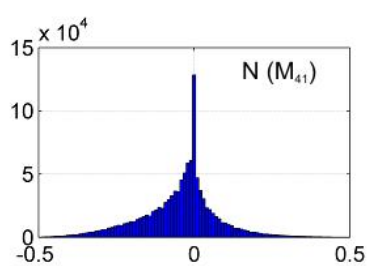

(3)

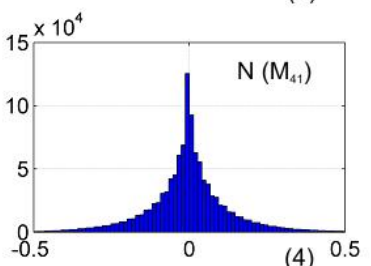

Fig. 4. Coordinate distributions of the Mueller-matrix rotation invariant $M_{41}$ for optically thin histological sections of postsurgical biopsy of benign (fragments (1), (3)) and malignant (fragments (2), (4)) tumor of uterus wall.
Let us analyze the obtained results from the physical point of view. The samples of both types within the framework of considered model (Eqs. (1)-(8)) represent the systems of linear $\left(F_{12 ; 21 ; 22 ; 33}(a, b)\right)$ and elliptic $\left(F_{44}(c)\right)$ fluorescent oscillators in the opticallyanisotropic matrix with linear Eq. (6) and circular Eq. (7) birefringence.

As it is well known [21-31], orientationally ( $\rho$ )phase $(\delta, \theta)$ structure of this matrix depends on physiological (pathological) state of biological tissue. More disordered by directions $(\Delta \rho \uparrow)$ birefringent ( $\Delta n \approx$ const) fibrillar network is typical for malignant states. On the other hand, in [29-31] it was shown that autofluorescence in the red spectral range being increased together with growth and development of tumor. This phenomenon can be related with the liquidcrystal networks of porphyrins, which accumulate in tissues of malignant new formations on different stages of their development.

Thus, the malignant states are accompanied by formation of prevailed, over the disordered linear oscillators, system of fluorescent elliptical oscillators. Vice-versa, in the case of pre-cancer state the prevailing of linear fluorescent oscillators is more typical. In other words, for tissue with dysplasia the following analytical scenario is realized:

$\left\{\begin{array}{l}a \uparrow, b \uparrow \rightarrow F_{12 ; 21 ; 22 ; 33}(a, b) \uparrow \Rightarrow M_{14} \Uparrow, \\ c \downarrow \rightarrow F_{44}(c) \downarrow \Rightarrow M_{41} \Downarrow .\end{array}\right.$

The inverse regularity takes place for malignant new formations:

$\left\{\begin{array}{l}a \downarrow, b \downarrow \rightarrow F_{12 ; 21 ; 22 ; 33}(a, b) \downarrow \Rightarrow M_{14} \Downarrow, \\ c \uparrow \rightarrow F_{44}(c) \uparrow \Rightarrow M_{41} \Uparrow .\end{array}\right.$

The secondary phase modulation of fluorescent radiation by fibrillar networks of both types samples does not introduce any sufficient changes in the mentioned scenario. As far as for pre-cancer and cancer states, it is typical practically the same birefringence $(\Delta n \approx$ const) of protein structures.

The results of the quantitative statistical (relations (11)) analysis of the series of Mueller-matrix rotation invariants of both groups of histological sections are illustrated by the data presented in Table 1.

Table 1. Statistical $\left(Z_{i=1 ; 2 ; 3 ; 4}\right)$ moments of the $1^{\text {st }}$ to $4^{\text {th }}$ orders distribution of Mueller-matrix invariants of histological sections of uterus wall tumor.

\begin{tabular}{|c|c|c|c|c|}
\hline \multirow[b]{2}{*}{ 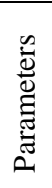 } & \multicolumn{2}{|c|}{$M_{14}$} & \multicolumn{2}{|c|}{$M_{41}$} \\
\hline & $\begin{array}{l}\frac{\pi}{\frac{\pi}{3}} \\
\frac{\pi}{2} \\
\frac{0}{0} \\
\hat{n}\end{array}$ & 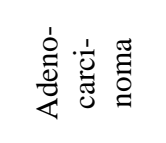 & $\frac{\frac{\pi}{\tilde{a}}}{\frac{\pi}{2}}$ & 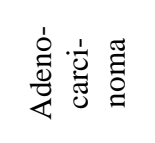 \\
\hline$Z_{1}$ & $0.19 \pm 0.023$ & $0.09 \pm 0.012$ & $0.055 \pm 0.01$ & $0.13 \pm 0.019$ \\
\hline$Z_{2}$ & $0.23 \pm 0.039$ & $0.12 \pm 0.017$ & $0.11 \pm 0.015$ & $0.21 \pm 0.036$ \\
\hline$Z_{3}$ & $0.73 \pm 0.11$ & $1.81 \pm 0.24$ & $2.87 \pm 0.43$ & $0.94 \pm 0.14$ \\
\hline$Z_{4}$ & $1.12 \pm 0.17$ & $2.18 \pm 0.31$ & $3.29 \pm 0.53$ & $1.62 \pm 0.27$ \\
\hline
\end{tabular}


Table 2. Sensitivity and specificity of Mueller-matrix technique of mapping laser polarization fluorescence in the samples of uterus wall tumor.

\begin{tabular}{|c|c|c|}
\hline Parameters & $M_{14}$ & $M_{41}$ \\
\hline$S e\left(Z_{i}\right), \%$ & 80 & 93 \\
\hline$S p\left(Z_{i}\right), \%$ & 75 & 85 \\
\hline
\end{tabular}

The following quantitative criteria of differentiation of benign and malignant changes were determined due to the analyzed mechanisms of laser polarization autofluorescence:

- the Mueller-matrix image $M_{14}(m \times n)$ of a histological section of benign tumor is characterized by greater values of statistical moments of the $1^{\text {st }} Z_{1}\left(M_{14}\right)(2.1$ times $)$ and $2^{\text {nd }}$ $Z_{2}\left(M_{14}\right)$ (2 times) orders. For statistical moments of higher orders, the opposite tendency is typical $Z_{3}\left(M_{14}\right) \quad(2.5$ times decrease $)$ and $Z_{4}\left(M_{14}\right)$ (2.4 times decrease).

- $\quad$ statistically third-order structures of polypeptide chains of collagen and myosin can be differentiated by the following properties: $Z_{1}\left(M_{41}\right)$ - increase by 2.4 times; $Z_{2}\left(M_{41}\right)$ - increase by 1.95 times; $Z_{3}\left(M_{41}\right)$ - decrease by 3 times and $Z_{4}\left(M_{41}\right)$ decrease by 2 times.

Within both groups of histological sections, by statistical approaches the sensitivity $S e=\frac{d}{d+p} 100 \%$ and specificity $S p=\frac{u}{u+h} 100 \%$ of the technique of Mueller-matrix mapping of laser polarization fluorescence observed in protein networks were determined, where $d$ and $p$ are the amounts of right and wrong diagnoses within the group $1 ; u$ and $h-$ the same within group 2.

For different Mueller-matrix rotation invariants of laser polarization autofluorescence, the following results were obtained (Table 2).

Thus, the statistical analysis of spectral-selective Mueller-matrix fluorescent invariants proved to be efficient when solving the task of differential diagnostics of benign and malignant changes in uterus wall.

\section{Conclusions}

1. The model of laser polarization autofluorescence of biological tissues has been suggested in which the mechanisms of optical anisotropic absorption, autofluorescence and birefringence have been taken into consideration.

2. The interrelations between statistical parameters characterizing spectral-selective Mueller-matrix fluorescent images and the peculiarities of the mechanisms of porphyrin fluorescence in optically anisotropic histological sections of uterus wall biopsy have been found.
3. The efficiency of the method of azimuthally invariant spectral-selective Mueller-matrix mapping of laser polarization autofluorescence of protein networks in the task of differentiation of benign and malignant tumors of uterus wall has been demonstrated.

\section{Acknowledgement}

This work was supported by the grants № 0113U003239 and № 0112 U002336 from the Ukrainian Foundation for Basic Researches.

\section{References}

1. T.T. Tower, R.T. Tranquillo, Alignment maps of tissues: I. Microscopic elliptical polarimetry // Biophys. J. 81, p. 2954-2963 (2001).

2. M.H. Smith, P. Burke, A. Lompado, E. Tanner, L.W. Hillman, Mueller matrix imaging polarimetry in dermatology // Proc. SPIE, 3991, p. 210-216 (2000).

3. M. Shribak, R. Oldenbourg, Techniques for fast and sensitive measurements of two-dimensional birefringence distributions // Appl. Opt. 42, p. 3009-3017 (2003).

4. M.H Smith, Interpreting Mueller matrix images of tissues // Proc. SPIE, 4257, p. $82-89$ (2001).

5. X. Wang, L.V. Wang, Propagation of polarized light in birefringent turbid media: A Monte Carlo study // J. Biomed. Opt. 7, p. 279-290 (2002).

6. O.V. Angelsky, A.Ya. Bekshaev, A.Ya. Maksimyak, P.P. Maksimyak, I.I. Mokhun, S.G. Hanson, C.Yu. Zenkova, A.V. Tyurin, Circular motion of particles suspended in a Gaussian beam with circular polarization validates the spin part of the internal energy flow // Opt. Exp. 20, p. 1135111356 (2012).

7. A.Ya. Bekshaev, O.V. Angelsky, S.G. Hanson, C.Yu. Zenkova, Scattering of inhomogeneous circularly polarized optical field and mechanical manifestation of the internal energy flows // Phys. Rev. A, 86, 023847 (2012).

8. O.V. Angelsky, P.P. Maksimyak, T.O. Perun, Dimensionality in optical fields and signals // Appl. Opt. 32, p. 6066-6071 (1993).

9. O.V. Angelsky, Y.A. Ushenko, The degree of mutual anisotropy of biological liquid crystals net during the diagnostics of human tissues birefringence // Adv. Opt. Technol. 2010, 321275 (2010)

10. O.V. Angelsky, A.Ya. Bekshaev, P.P. Maksimyak, A.P. Maksimyak, S.G. Hanson, C.Yu. Zenkova, Orbital rotation without orbital angular momentum: mechanical action of the spin part of the internal energy flow in light beams // Opt. Exp. 20(4), p. 3563-3571 (2012). 
11. M.P. Bard, A. Amelink, M. Skurichina et al., Optical spectroscopy for the classification of malignant lesions of the bronchial tree // Chest. 129, p. 995-1001 (2006).

12. J.F. Beamis A. Ernst, P. Mathur, R. Yung, M. Simoff, A multi-center study comparing autofluorescence bronchoscopy to white light bronchoscopy // Lung Cancer, 41, p. S49-S50 (2003).

13. K. Häussinger, J. Pichler, F. Stanzel, A. Markus, H. Stepp, A. Morresi-Hauff, R. Baumgartner, Autofluorescence bronchoscopy: the D-light system // Intervent. Bronchoscopy, 30, p. 243-252 (2000).

14. F. Herth, H. Becker, Autofluorescence bronchoscopy-a comparison of two systems (LIFE and D-Light) // Respiration, 70, p. 395-398 (2003).

15. J. Hung, S. Lam, J.C. Leriche, B. Palcic, Autofluorescence of normal and malignant bronchial tissue // Lasers Surg. Med. 11, p. 99-105 (1991).

16. A.V. Kamath, P.N. Chhajed, Role of bronchoscopy in early diagnosis of lung cancer // Indian J. Chest Dis. Allied Sci. 48, p. 265-269 (2006).

17. L.I. Grossweiner, A. Blum, G.C. Goyal, Advances in Experimental Medicine and Biology. Methods in Porphyrin Photosensitization, 193, p. 181-192 (1985).

18. S.N. Savenkov, V.V. Marienko, E.A. Oberemok, O.I. Sydoruk, Generalized matrix equivalence theorem for polarization theory // Phys. Rev. E, 74, p. 605-607 (2006).

19. O. Arteaga, S. Nichols, B. Kahr, Mueller matrices in fluorescence scattering // Opt. Lett. 37, p. 2835 2837 (2012).

20. R. Alfano, D. Tata, J. Cordero et al., Laser induced fluorescence spectroscopy from native cancerous and normal tissue // IEEE Quantum Electron. 20, p. 1502 (1984).

21. A.G. Ushenko, V.P. Pishak, Laser polarimetry of biological tissue: Principles and applications, in: Handbook of Coherent-Domain Optical Methods: Biomedical Diagnostics, Environmental and Material Science, 1, p. 93-138 (2004).

22. .O.V. Angelsky, A.G. Ushenko, Yu.A. Ushenko, V.P. Pishak, A.P. Peresunko, Statistical, correlation and topological approaches in diagnostics of the structure and physiological state of birefringent biological tissues, in: Handbook of Photonics for Biomedical Science, p. 283-322, 2010.

23. Y.A. Ushenko, T.M. Boychuk, V.T. Bachynsky, O.P. Mincer, Diagnostics of structure and physiological state of birefringent biological tissues: Statistical, correlation and topological approaches, in: Handbook of Coherent-Domain Optical Methods, p. 107-148 (2013).

24. Yu.A. Ushenko, G.B. Bodnar, G.D. Koval, Classifying optical properties of surface-and bulkscattering biological layers with polarization singular states // J. Innov. Opt. Health Sci. 6, 1350018 (2013).

25. Yu.A. Ushenko, Statistical structure of polarization-inhomogeneous images of biotissues with different morphological structures // Ukr. J. Phys. Opt. 6, p. 63-70 (2005).

26. Yu.A. Ushenko, A.P. Peresunko, B.A. Baku, A new method of Mueller-matrix diagnostics and differentiation of early oncological changes of the skin derma // Adv. Opt. Technol. 952423 (2010).

27. Yu.A. Ushenko, O.I. Telenga, A.P. Peresunko, O.K. Numan, New parameter for describing and analyzing the optical-anisotropic properties of biological tissues // J. Innov. Opt. Health Sci. 4 p. 463-475 (2011).

28. Yu.A. Ushenko, The feasibilities of using the statistical, fractal and singular processing of hominal blood plasma phase images during the diagnostics and differentiation of mammary gland pathological states // J. Innov. Opt. Health Sci. 5, 1150001 (2012).

29. R. Sroka, R. Baumgartner, A. Buser, C. Ell, D. Jocham, E. Unsoeld, Laser assisted detection of endogenous porphyrin in malignant diseases // Proc. SPIE, 1641, p. 99-105 (1991).

30. M.A. D'Hallewin, A.R. Kamuhabwa, T. Roskams, P.A. De Witte, L. Baert, Hypericin based fluorescence diagnosis of bladder carcinoma // BJU Int. 89, p. 760-763 (2002).

31. M.A. D’Hallewin, L. Bezdetnaya, F. Guillemin, Fluorescence detection of bladder cancer: a review // Eur. Urol. 42, p. 417-425 (2002). 